\title{
Estructuras Neurovasculares en Riesgo Durante Abordajes Artroscópicos en Tobillo. Un Estudio Cadavérico
}

\author{
Neurovascular Structures at Risk During Arthroscopic \\ Approaches to the Ankle. A Cadaveric Study
}

\author{
Alejandra Nohemí Hernández-Rodríguez*; Rodolfo Morales-Avalos*; Salvador Galindo de-León*; \\ María del Carmen Theriot-Giron"; Félix Vilchez-Cavazos"**; Rodrigo E. Elizondo-Omaña* \& Santos Guzmán-López
}

HERNÁNDEZ-RODRÍGUEZ, A. N.; MORALES-AVALOS, R.; DE-LEÓN, S. G.; THERIOT-GIRON, M. C.; VILCHEZCAVAZOS, F.; ELIZONDO-OMAÑA, R. E. \& GUZMÁN-LÓPEZ, S. Estructuras neurovasculares en riesgo durante abordajes artroscópicos en tobillo. Un estudio cadavérico. Int. J. Morphol., 34(4):1308-1312, 2016.

RESUMEN: La artroscopía de tobillo ha tenido un aumento en su utilización en las últimas tres décadas para la corrección quirúrgica de afecciones del tobillo. Se prefiere a la cirugía abierta por sus múltiples ventajas, destacando la disminución de las complicaciones, con una prevalencia de 7,3\% para la artroscopia y 15,9\% para la cirugía abierta. Estudios previos de artroscopia de tobillo reportan complicaciones generales entre el 0,9 a $17 \%$, de las cuales el 33 a $50 \%$ envuelven los nervios cutáneos, principalmente el nervio fibular superficial o alguna de sus ramas. El objetivo del presente estudio fue determinar estructuras neurovasculares en riesgo próximas a los portales artroscópicos del tobillo descritos en la literatura. Se llevó a cabo un estudio anatómico observacional, transversal y descriptivo. La muestra consistió en 10 tobillos de población mexicana en los cuales se introdujeron cánulas artroscópicas en algunos portales de tobillo descritos en la literatura y se realizó una disección superficial de los pies, posteriormente se determinó la distancia entre las estructuras neurovasculares próximas a los portales y se realizó un análisis estadístico con los resultados. Los resultados del estudio anatómico evidenció que portal antero-medial es el portal más seguro debido a que la distancia de dicho portal a una estructura neurovascular fue la más elevada, obteniendo una media de $11,30 \mathrm{~mm} \pm 11,25$, la menor distancia encontrada fue la del portal posteromedial con una media de 2,84 mm $\pm 1,28$. El $10 \%$ de los portales laterales resultaron con lesión de estructuras venosas tributarias de la vena safena menor. El portal antero-medial es el portal más seguro pero con mayor variabilidad respecto a las distancias de las estructuras neurovasculares al portal y el portal postero-medial es el de mayor riesgo debido a la mayor presencia de estructuras neurovasculares.

PALABRAS CLAVE: Artroscopia; Tobillo; Estructuras en riesgo; Nervios cutáneos; Anatomía; Cadáver.

\section{INTRODUCCIÓN}

La artroscopia de tobillo ha tenido un aumento en su utilización en las últimas tres décadas para la corrección de afecciones del tobillo, tales como la compresión de hueso y tejidos blandos, lesiones osteocondrales, artrodesis de tobi1lo, inestabilidad del tobillo, artritis séptica, artrofibrosis, osteoartrosis, sinovitis y fracturas (Glazebrook et al., 2009).

La artroscopia se prefiere a la cirugía abierta por las ventajas que conlleva su realización, entre éstas destaca la disminución de las complicaciones durante el procedimiento y el post operatorio, con una prevalencia de 7,3\% para la artroscopia y 15,9\% para la cirugía abierta (Zwiers et al., 2013).
Estudios clínicos previos acerca de artroscopia de tobillo reportan complicaciones generales entre el 0,9 a 17 $\%$, de las cuales el 33 a $50 \%$ involucran la lesión de los nervios cutáneos del pie y el tobillo, principalmente el nervio fibular superficial o una de sus ramas (Duscher et al., 2014).

Existen reportes de casos de lesiones del nervio fibular superficial durante la introducción de los portales en la artroscopia de tobillo (Takao et al., 2001). Se ha reportado la formación de pseudoaneurismas de la arteria tibial anterior, posterior a la intervención (Mariani et al., 2001) y otras complicaciones como lo son lesiones vasculares y

\footnotetext{
* Departamento de Anatomía Humana, Facultad de Medicina, U.A.N.L., Monterrey, Nuevo León, México.

** Departamento de Anatomía Humana, Facultad de Odontología, U.A.N.L., Monterrey, Nuevo León, México.

**** Servicio de Ortopedia y Traumatología, Hospital Universitario “Dr. José Eleuterio González”, U.A.N.L., Monterrey, Nuevo León, México.
} 
tendinosas, artritis séptica, tromboembolia y lesiones iatrogénicas (lesión de estructuras articulares) (Giannoudis et al., 2012).

La literatura evidencia que los nervios cutáneos del pie son las estructuras más frecuentemente lesionadas durante la introducción de los portales en la artroscopia de tobillo y que la localización de los mismos cambia en los pies de diferentes poblaciones, por lo que resulta importante analizar la seguridad de los portales respecto a las estructuras nerviosas (Lui \& Chan, 2010).

Se han descrito diferentes portales en la literatura, en el presente estudio se analizaron 6 de los más frecuentemente descritos. Dos anteriores (antero-medial y antero-lateral), dos posteriores (postero-medial y postero-lateral) y dos portales laterales (el del seno del tarso y un portal localizado 1 centímetro proximal y 1 centímetro posterior al maléolo lateral).

Las estructuras en riesgo de lesión varían en cada portal artroscópico en el tobillo. En el portal antero medial dicha estructura es el nervio safeno, mismo que se ha reportado que tiene una trayectoria más variable por lo que su estudio anatómico es de gran importancia. En el portal antero-lateral el nervio más cercano es el fibular superficial, el cual se ha reportado como la estructura más frecuentemente lesionada durante una artroscopia de tobillo. El nervio sural le sigue en frecuencia y la colocación del portal postero-lateral es el que podría lesionar dicho nervio (Duscher et al.). El portal postero-medial ha sido estudiado abundantemente debido a que está en extrema cercanía con el túnel del tarso, se ha reportado que la estructura más cercana al portal es el nervio tibial posterior (Sora et al., 2008).

No se encontró un estudio anatómico en la población mexicana, lo que nos lleva a profundizar en el tema para encontrar los portales con mayor riesgo de lesión neurovascular y poder seleccionar los portales más seguros. El objetivo de este estudio fue determinar las estructuras neurovasculares en riesgo de lesión próximas a los portales artroscópicos de tobillo descritos en la literatura.

\section{MATERIAL Y MÉTODO}

Estudio anatómico, observacional, transversal, descriptivo. Se analizaron 10 tobillos ( 5 cadáveres embalsamados) pertenecientes a los Departamentos de Anatomía Humana de la Facultades de Medicina y Odontología de la Universidad Autónoma de Nuevo León. Los tobillos utilizados estaban exentos de traumatismos o signos de cirugías previas en la extremidad inferior. Los cadáveres se colocaron en posición de decúbito supino para la colocación de los portales anteriores y laterales y en posición de decúbito prono para la colocación de los portales posteriores con los pies en posición neutral (Fig. 1). Se utilizaron cánulas artroscópicos de 4 milímetros de diámetro para la colocación de los portales.

Para la colocación de los portales se utilizaron referencias anatómicas descritas en la literatura y se introdujeron todos al nivel de la articulación del tobillo. Se comenzó con el portal anteromedial (descrito como el portal más seguro) (Ferkel et al., 1996). Se tomó de referencia el tendón del músculo tibial anterior y el portal se introdujo medial a éste, en el portal anterolateral la referencia anatómica es el tendón del músculo extensor común de los dedos y el portal se introdujo lateral a éste (Hammond et al., 2011). El portal lateral del seno del tarso es usado como distracción para acceder a la articulación del tobillo y es introducido a base de la palpación del seno del tarso (Beimers et al., 2009). El portal lateral $1 \mathrm{~cm}-1 \mathrm{~cm}$ es introducido 1 centímetro proximal y 1 centímetro posterior al vértice del maléolo lateral (Lee et al., 2008). Los dos portales posteriores comparten el tendón calcáneo como referencia anatómica para su colocación, el portal posteromedial se introduce medial al tendón calcáneo y lateral al tendón del músculo del flexor largo del primer dedo por tener un paquete neurovascular de importancia en dirección medial a éste. El portal posterolateral se introdujo lateral al tendón calcáneo (Lee et al.).

Posteriormente se llevó a cabo la disección en un plano superficial comenzando con la piel y tejido subcutáneo (Fig. 2A) se examinaron las estructuras próximas a los portales y
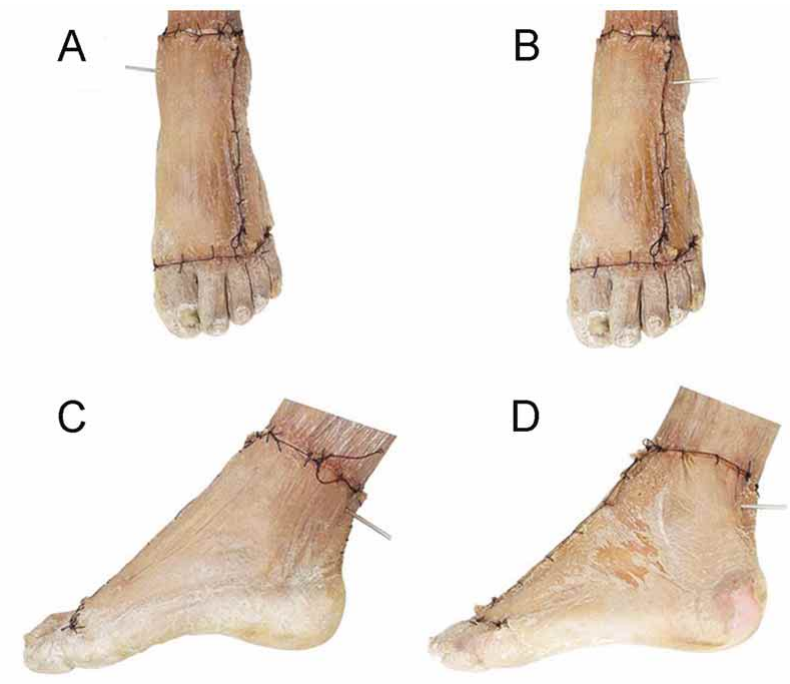

Fig. 1. Técnica de colocación de portales artroscopicos en el tobillo. A) Portal antero-medial. B) Portal antero-lateral. C) Portal postero-medial. D) Portal postero-lateral. 
HERNÁNDEZ-RODRÍGUEZ, A. N.; MORALES-AVALOS, R.; DE-LEÓN, S. G.; THERIOT-GIRON, M. C.; VILCHEZ-CAVAZOS, F.; ELIZONDO-OMAÑA, R. E. \& GUZMÁN-LÓPEZ, S. Estructuras neurovasculares en riesgo durante abordajes artroscópicos en tobillo. Un estudio cadavérico. Int. J. Morphol., 34(4):1308-1312, 2016.

se midió la distancias de los portales a dichas estructuras con un vernier digital milimétrico con una precisión de 0.01 milímetros (Mitutoyo Digimatic w/Absolute Encoders Series 500) (Fig. 2B). Se evaluó el porcentaje de lesión de estructuras nerviosas y vasculares ocurridas en el presente estudio.
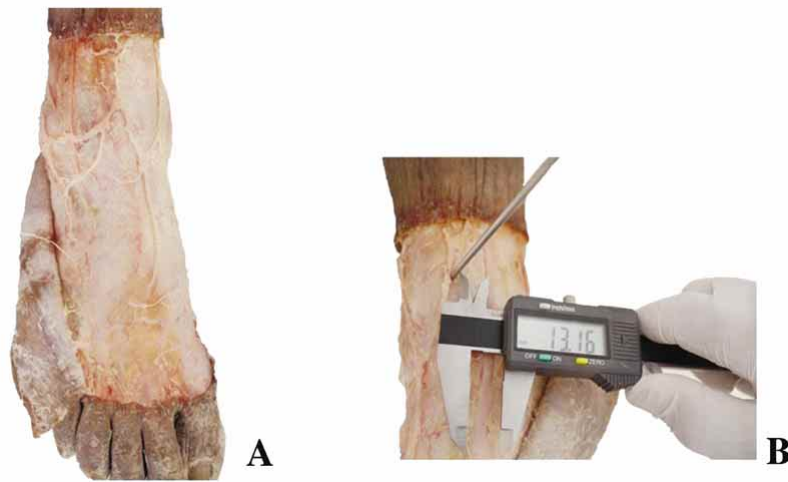

Fig. 2. Fotografías representativas de las disecciones realizadas en el presente estudio. A) Disección superficial. B) Técnica de medición de las estructuras.

Análisis Estadístico. Se realizó un análisis estadístico de las mediciones utilizando en el programa computacional SPSS Versión 21.0 (Chicago, IL). En donde se calculó la media y desviación estándar de las distancias entre el portal y las estructuras neurovasculares más próximas así como el límite inferior, límite superior y el intervalo de confianza al $95 \%$.

Consideraciones Éticas: El presente protocolo fue aprobado por el Comité de Ética y Comité de Investigación de la Facultad de Medicina de la U.A.N.L. con el número de registro AH13-009. No existieron ganancias financieras o comerciales por la realización de este estudio, por lo que los autores declaran no tener conflictos de intereses.

\section{RESULTADOS}

En el presente estudio se hizo énfasis en medir la distancia de cada uno de los portales con su estructura neurovascular más cercana, obteniendo posteriormente la media y la desviación estándar de cada uno de los diez tobillos, además se determinó el porcentaje de lesión nerviosa o vascular encontrada.

La distancia entre el portal antero- medial y el nervio safeno obtuvo una media de 11,30 mm $\pm 11,25$ (Tabla I).

La distancia promedio del portal antero-lateral al nervio fibular superficial fue de $6,58 \mathrm{~mm} \pm 4,08$ (Tabla I).

La media del portal postero-medial al nervio tibial posterior 2,8 $\mathrm{mm} \pm 1,28$, siendo ésta la menor distancia de todas reportadas en el estudio. Del portal postero-lateral al nervio sural se obtuvo 4,24 mm $\pm 2,29$ (Tabla I).

En los portales laterales, las estructuras más cercanas fueron tributarias de la vena safena menor, en el portal del seno del tarso la media de la distancia a la estructura más cercana $3,82 \mathrm{~mm} \pm 2,64$, en cuanto al portal $1 \mathrm{~cm}$ proximal y $1 \mathrm{~cm}$ posterior al maléolo lateral la media fue de 2,01 $\mathrm{mm} \pm 1,71$ (Tabla II).

Se evaluó el porcentaje de lesión y se obtuvo un $10 \%$ de lesión venosa en su mayoría en los portales laterales que corresponde el $3 \%$ al portal del seno del tarso, $3 \%$ al portal $1 \mathrm{~cm}$ proximal y $1 \mathrm{~cm}$ posterior al maléolo lateral, $2 \%$ pertenece al portal postero-lateral y otro $2 \%$ al portal antero-lateral.

Tabla I. Distancia promedio de portales evaluados con las estructuras nerviosas superficiales analizadas en el presente estudio.

\begin{tabular}{llccc}
\hline \multicolumn{1}{c}{ Portal } & \multicolumn{1}{c}{ Estructura nerviosa } & $\begin{array}{c}\text { Media y Desviación } \\
\text { estándar } \\
(\mathrm{mm})\end{array}$ & $\begin{array}{c}\text { 95\% Intervalo de confianza } \\
\text { Límite Inferior } \\
(\mathrm{mm})\end{array}$ & $\begin{array}{c}\text { Límite superior } \\
(\mathrm{mm})\end{array}$ \\
\hline Antero-medial & N. Safeno & $11,30 \pm 11,25$ & 3,25 & 19,35 \\
Antero-lateral & N. Fibular superficial & $6,58 \pm 4.08$ & 3,43 & 9,72 \\
Postero-medial & N. Tibial posterior & $2,84 \pm 1.28$ & 1,86 & 3,83 \\
Postero-lateral & N. Sural & $4,24 \pm 2.29$ & 2,47 & 6,00 \\
\hline
\end{tabular}

Tabla II. Distancia de Portales laterales a estructuras vasculares.

\begin{tabular}{lcccc}
\hline Portal & Estructura venosa & $\begin{array}{c}\text { Media y desviación } \\
\text { estándar } \\
(\mathrm{mm})\end{array}$ & $\begin{array}{c}95 \% \text { Intervalo de confianza } \\
\text { Límite inferior } \\
(\mathrm{mm})\end{array}$ & $\begin{array}{c}\text { Límite superior } \\
(\mathrm{mm})\end{array}$ \\
\hline Seno del tarso & Tributarias de & $3,82 \pm 2,64$ & 1,93 & 5,72 \\
$1 \mathrm{~cm}$ proximal y $1 \mathrm{~cm}$ posterior & Tributarias de & $2,01 \pm 1,71$ & 0,69 & 3,33 \\
\hline
\end{tabular}




\section{DISCUSIÓN}

Diversos estudios han reportado las complicaciones clínicas de la artroscopia de tobillo debido a que dicho procedimiento se ha convertido en la técnica invasiva estándar para diagnóstico y tratamiento de las afecciones del tobillo (Takao et al.).

En la literatura se reporta en un estudio que la media del portal antero-medial con el nervio safeno fue de $12 \mathrm{~mm}$ (Lui \& Chan). Resultado que no difiere mucho con nuestro estudio, obtuvimos una media de $11,30 \mathrm{~mm} \pm 11,25$, la desviación estándar alta nos muestra que es también el nervio que más variabilidad tiene, como ya se ha reportado en la literatura (Duscher et al.).

Del portal antero-lateral se reporta una media de $4 \mathrm{~mm}$ al nervio fibular superficial (Lui \& Chan). En nuestro estudio obtuvimos una media mayor $6,58 \mathrm{~mm} \pm 4,08$.

De los portales posteriores el más estudiado ha sido el portal postero-medial por la cercanía al túnel del tarso. Estudios han reportado diferentes distancias en diversas poblaciones (Tabla III).

En nuestro estudio la media del portal postero-medial al nervio tibial posterior fue de $2,84 \mathrm{~mm} \pm 1,28$, siendo la menor distancia reportada en la literatura y considerándose como el portal de mayor riesgo de lesionar nervio.

El portal postero-lateral y sus estructuras en riesgo también han sido de los más estudiados, se ha reportado la laceración parcial del nervio sural en un espécimen (Mouilhade et al., 2011). En la literatura se encuentran distancias del portal al nervio sural de 13,1 mm $\pm 3,0$ (Urgüden et al., 2009). En el presente estudio no se reportó lesión del nervio sural en el portal postero-lateral y la distancia fue menor de las reportadas en la literatura con 4,24 $\mathrm{mm} \pm 2,29$.

Los portales del seno del tarso, el $1 \mathrm{~cm}$ proximal y $1 \mathrm{~cm}$ posterior al maleolo lateral, el antero lateral y postero lateral en nuestro estudio se reportó un $10 \%$ de lesión de venas tributarias de la safena menor, en un estudio se ha descrito que no había riesgo de lesionar ninguna estructura neurovascular (Hammond et al.).

Tabla III. Resultados de diversos estudios para la distancia del portal postero-medial al nervio tibial posterior.

\begin{tabular}{ll}
\hline Población/ Estudio/ Año & \multicolumn{1}{c}{ Distancia } \\
\hline Francia. Moulhade et al. (2011) & $6,8 \mathrm{~mm}(4,4-9,2)$ \\
Estados Unidos. Feiwell \& Frey (1993) & $7,5 \mathrm{~mm}$ \\
Italia. Lijoi et al. (2003) & $14,7 \mathrm{~mm}(11-17 \mathrm{~mm})$ \\
Turquía. Urgüden et al. (2009) & $16,5 \mathrm{~mm} \pm 5,6 \mathrm{~mm}$ \\
Mexicana. Hernández et al. (2015) & $2,84 \mathrm{~mm} \pm 1,28$ \\
(Presente Estudio) & \\
\hline
\end{tabular}

HERNÁNDEZ-RODRÍGUEZ, A. N.; MORALESAVALOS, R.; DE-LEÓN, S. G.; THERIOT-GIRON, M. C.; VILCHEZ-CAVAZOS, F.; ELIZONDO-OMAÑA, R. E. \& GUZMÁN-LÓPEZ, S. Estructuras neurovasculares en riesgo durante abordajes artroscópicos en tobillo. Un estudio cadavérico. Int. J. Morphol., 34(4):1308-1312, 2016.

SUMMARY: In the last three decades the procedure of ankle arthroscopy has increased in ankle surgery. It is preferred to open surgery for multiple reasons, the most important is that it has fewer complications, with a prevalence of $7.3 \%$ for arthroscopy and $15.9 \%$ for open surgery. Previous studies of ankle arthroscopy reported general complications between 0.9 to $17 \%$ which involved cutaneous nerves at a rate of 33 to $50 \%$, mainly the superficial peroneal nerve. The principal objective of this study was to determine the neurovascular structures near the arthroscopic portals of the ankle. We carried out anatomical, observational, transversal and descriptive studies. This study was performed with 10 ankles of Mexican population. A $4 \mathrm{~mm}$ trocar was introduced in some of the portals described in the literature and superficial dissection of the feet was made. Subsequently, the distance between the nearest neurovascular structures was measured, and statistical analysis of the results was realized. The results of the anatomic study was that the anterior-medial portal was considered the safest portal because it has the highest distance between the portal and the neurovascular structure, with an average of $11.30 \mathrm{~mm} \pm 11.25$, the posterior-medial portal has the smallest average with 2.84 $\mathrm{mm} \pm 1.28$. A lesion of the small saphenous vein was reported in $10 \%$ of the lateral portals. The antero-medial portal is the safest but with highest variability regarding the distances of the neurovascular structures to the portal and the posteromedial portal has the highest risk due to the increased presence of neurovascular structures.

KEY WORDS: Arthroscopy; Ankle; Structures at risk; Cutaneous Nerves; Anatomy.

\section{REFERENCIAS BIBLIOGRAFICAS}

Beimers, L.; de Leeuw, P. A. \& van Dijk, C. N. A 3-portal approach for arthroscopic subtalar arthrodesis. Knee Surg. Sports Traumatol. Arthrosc., 17(7):830-4, 2009.

Duscher, D.; Wenny, R.; Entenfellner, J.; Weninger, P. \& Hirtler, L. Cutaneous innervation of the ankle: an anatomical study showing danger zones for ankle surgery. Clin. Anat., 27(4):653-8, 2014.

Feiwell, L. A. \& Frey, C. Anatomic study of arthroscopic portal sites of the ankle. Foot Ankle, 14(3):142-7, 1993.

Ferkel, R. D.; Heath, D. D. \& Guhl, J. F. Neurological complications of ankle arthroscopy. Arthroscopy, 12(2):200-8, 1996. 
HERNÁNDEZ-RODRÍGUEZ, A. N.; MORALES-AVALOS, R.; DE-LEÓN, S. G.; THERIOT-GIRON, M. C.; VILCHEZ-CAVAZOS, F.; ELIZONDO-OMAÑA, R. E. \& GUZMÁN-LÓPEZ,

S. Estructuras neurovasculares en riesgo durante abordajes artroscópicos en tobillo. Un estudio cadavérico. Int. J. Morphol., 34(4):1308-1312, 2016.

Glazebrook, M. A.; Ganapathy, V.; Bridge, M. A.; Stone, J. W. \& Allard, J. P. Evidence-based indications for ankle arthroscopy. Arthroscopy, 25(12):1478-90, 2009.

Hammond, A. W.; Phisitkul, P.; Femino, J. \& Amendola, A. Arthroscopic debridement of the talonavicular joint using dorsomedial and dorsolateral portals: a cadaveric study of safety and access. Arthroscopy, 27(2):228-34, 2011.

Giannoudis, P. V. (Ed.). Practical Procedures in Elective Orthopaedic Surgery. London, Springer, 2012.

Lee, K. B.; Saltzman, C. L.; Suh, J. S.; Wasserman, L. \& Amendola, A. A posterior 3-portal arthroscopic approach for isolated subtalar arthrodesis. Arthroscopy, 24(11):1306-10, 2008.

Lijoi, F.; Lughi, M. \& Baccarani, G. Posterior arthroscopic approach to the ankle: an anatomic study. Arthroscopy, 19(1):62-7, 2003.

Lui, T. H. \& Chan, L. K. Safety and efficacy of talonavicular arthroscopy in arthroscopic triple arthrodesis. A cadaveric study. Knee Surg. Sports Traumatol. Arthrosc., 18(5):607-11, 2010.

Mariani, P. P.; Mancini, L. \& Giorgini, T. L. Pseudoaneurysm as a complication of ankle arthroscopy. Arthroscopy, 17(4):400-2, 2001.

Mouilhade, F.; Oger, P.; Roussignol, X.; Boisrenoult, P.; Sfez, J. \& Duparc, F. Risks relating to posterior 2-portal arthroscopic subtalar arthrodesis and articular surfaces abrasion quality achievable with these approaches: a cadaver study. Orthop. Traumatol. Surg. Res., 97(4):396-400, 2011.

Sora, M. C.; Jilavu, R.; Grübl, A.; Genser-Strobl, B.; Staykov, D. $\&$ Seicean, A. The posteromedial neurovascular bundle of the ankle: an anatomic study using plastinated cross sections. Arthroscopy, 24(3):258-263.e1, 2008.

Takao, M.; Ochi, M.; Shu, N.; Uchio, Y.; Naito, K.; Tobita, M.; Matsusaki, M. \& Kawasaki, K. A case of superficial peroneal nerve injury during ankle arthroscopy. Arthroscopy, 17(4):4034, 2001 .

Urgüden, M.; Cevikol, C.; Dabak, T. K.; Karaali, K.; Aydin, A. T. \& Apaydin, A. Effect of joint motion on safety of portals in posterior ankle arthroscopy. Arthroscopy, 25(12):1442-6, 2009.

Zwiers, R.; Wiegerinck, J. I.; Murawski, C. D.; Smyth, N. A.; Kennedy, J. G. \& Van Djik, C. N. Surgical treatment for posterior ankle impingement. Arthroscopy, 29(7):1263-70, 2013.
Dirección para correspondencia:

Dr. C. Rodrigo E. Elizondo Omaña

Departamento de Anatomía Humana

Facultad de Medicina

Universidad Autónoma de Nuevo León (UANL)

Ave. Madero s/n Col. Mitras Centro

Monterrey, Nuevo León

C.P.64460

MÉXICO

E-mail: rodolfot59@hotmail.com

Recibido : 21-12-2015

Aceptado: 11-08-2016 